\title{
Increased expression of protease nexin-1 in fibroblasts during idiopathic pulmonary fibrosis regulates thrombin activity and fibronectin expression
}

Déborah François ${ }^{1,2}$, Laurence Venisse ${ }^{1,2}$, Joëlle Marchal-Somme ${ }^{2,3}$, Martine Jandrot-Perrus ${ }^{1,2}$, Bruno Crestani ${ }^{2,3,4,5}$, Véronique Arocas $^{1,2}$ and Marie-Christine Bouton ${ }^{1,2}$

Idiopathic pulmonary fibrosis (IPF) is a chronic diffuse lung disease characterized by an accumulation of excess fibrous material in the lung. Protease nexin-1 (PN-1) is a tissue serpin produced by many cell types, including lung fibroblasts. $\mathrm{PN}-1$ is capable of regulating proteases of both coagulation and fibrinolysis systems, by inhibiting, respectively, thrombin and plasminergic enzymes. PN-1 is thus a good candidate for regulating tissue remodeling occurring during IPF. We demonstrated a significant increase of PN-1 expression in lung tissue extracts, lung fibroblasts and bronchoalveolar lavage fluids of patients with IPF. The increase of PN-1 expression was reproduced after stimulation of control lung fibroblasts by transforming growth factor- $\beta$, a major pro-fibrotic cytokine involved in IPF. Another serpin, plasminogen activator inhibitor-1 (PAI-1) is also overexpressed in fibrotic fibroblasts. Unlike PAI-1, cell-bound PN-1 as well as secreted PN-1 from IPF and stimulated fibroblasts were shown to inhibit efficiently thrombin activity, indicating that both serpins should exhibit complementary roles in IPF pathogenesis, via their different preferential antiprotease activities. Moreover, we observed that overexpression of $\mathrm{PN}-1$ induced by transfection of control fibroblasts led to increased fibronectin expression, whereas $\mathrm{PN}-1$ silencing induced in fibrotic fibroblasts led to decreased fibronectin expression. Overexpression of PN-1 lacking either its antiprotease activity or its binding capacity to glycosaminoglycans had no effect on fibronectin expression. These novel findings suggest that modulation of PN-1 expression in lung fibroblasts may also have a role in the development of IPF by directly influencing the expression of extracellular matrix proteins. Our data provide new insights into the role of PN-1 in the poorly understood pathological processes involved in IPF and could therefore give rise to new therapeutic approaches.

Laboratory Investigation (2014) 94, 1237-1246; doi:10.1038/labinvest.2014.111; published online 8 September 2014

Idiopathic pulmonary fibrosis (IPF) is a chronic diffuse lung disease characterized by progressive deterioration in lung function ultimately leading to death. An important key event in IPF is excessive accumulation of extracellular matrix (ECM) components, resulting from an imbalance between matrix synthesis and degradation. Therefore the modulation of ECM proteolysis has a major regulatory role in the development of IPF and depends on the action of proteases, such as matrix metalloproteinases or proteases of the plasminergic system.

The control of protease activities is thus important in the homeostasis of the lung. Endogenous inhibitors, and in particular serine protease inhibitors (serpins), are known to have such a central role. Serpins form a large family of structurally related proteins present in the plasma or in tissues. They are suicide inhibitors using a unique and extensive conformational change to inhibit proteases. ${ }^{1}$ Among serpins, plasminogen activator inhibitor-1 (PAI-1) ${ }^{2,3}$ has been implicated in the pathophysiology of IPF. The level of plasminogen activation within the lung is indeed linked to the extent of fibrosis during IPF, as illustrated by the decrease in collagen accumulation observed after bleomycin-induced lung injury in mice with enhanced lung urokinase plasminogen activator (uPA) activity. ${ }^{4}$ We hypothesized that another serpin named protease nexin-1 (PN-1) or serpinE2, which is barely detectable in plasma but produced by most

\footnotetext{
${ }^{1}$ INSERM, U1148-LVTS, F-75018 Paris, France, Université Paris Diderot, Sorbonne Paris Cité, Paris, France; ${ }^{2}$ DHU FIRE, Paris, France; ${ }^{3}$ INSERM, U1152, F-75018 Paris, France, Université Paris Diderot, Sorbonne Paris Cité, Paris, France; ${ }^{4}$ Assistance Publique Hôpitaux de Paris, Hôpital Bichat, Service de Pneumologie A, Paris, France and ${ }^{5}$ Labex INFLAMEX, Paris, France

Correspondence: Dr M-C Bouton, PhD, Unité INSERM U1148-LVTS, CHU Xavier Bichat, 46 rue Henri Huchard, Paris Cedex 18 75877, France.

E-mail: marie-christine.bouton@inserm.fr

Received 3 March 2014; revised 3 July 2014; accepted 29 July 2014
} 
cell types, in particular human lung fibroblasts, ${ }^{5}$ might be a good candidate for the regulation of lung remodeling during IPF. Indeed, PN-1 is an inhibitor of uPA and tissue plasminogen activator but has a larger inhibition spectrum than PAI- 1 , as it is also able to efficiently inhibit thrombin and plasmin. ${ }^{6,7}$ Moreover, $\mathrm{PN}-1$ is thought to have a pathogenic role in the development of scleroderma, another fibrotic disease. ${ }^{8} \mathrm{PN}-1$ is thus a good candidate for regulating both ECM and fibrin deposition in tissues.

Although PN-1 was shown to be expressed in the normal adult mouse lung 9 and in the normal and pathological human lung, ${ }^{10}$ there have been no studies investigating the role of $\mathrm{PN}-1$ in pulmonary fibrosis. Our goal was thus to examine the expression of $\mathrm{PN}-1$ in lungs and in fibrotic human pulmonary fibroblasts from patients with IPF. Moreover, because transforming growth factor- $\beta$ (TGF- $\beta$ ) is a key pro-fibrotic cytokine implicated in IPF, known to regulate ECM production, cell growth, and tissue repair, ${ }^{11}$ we analyzed the regulation of $\mathrm{PN}-1$ expression by TGF- $\beta$ in pulmonary fibroblasts and the effect of such $\mathrm{PN}-1$ modulation on the behavior of these cells.

\section{MATERIALS AND METHODS Ethics Statement}

Human samples were used with the approval of the Institutional Review Board of the French learned society for respiratory medicine (Société de Pneumologie de Langue Française). Oral informed consent was obtained from the participants to the study at the time of bronchoscopy and recorded in the clinical charts. Clinical and functional data were collected on a standardized and anonymous collection form.

\section{Human Samples}

IPF was diagnosed according to the 2011 international statement. Bronchoalveolar lavages (BAL) were performed and lavage fluid processed as previously described. ${ }^{12}$ Fibrotic lung biopsies were derived from patients with IPF, and normal lung samples were obtained from a non-involved lung segment from patients undergoing lung surgery for a primary lung tumor.

\section{Immunohistochemistry}

Paraformaldehyde-fixed, paraffin-embedded sections of human lung tissue were used for immunohistochemical studies. The monoclonal anti-PN-1 IgG $(1 \mathrm{~F} 6 ; 4 \mu \mathrm{g} / \mathrm{ml})$ previously described, ${ }^{13}$ an anti- $\alpha$-smooth muscle actin $\operatorname{IgG}$ (Dako France SAS, Les Ulis, $4 \mu \mathrm{g} / \mathrm{ml}$ ), or an isotype-matched mouse IgG1 (Dako France SAS) antibody was used for immunohistochemistry. Positive staining was revealed using the Vectastain ABC-alkaline phosphatase kit mouse IgG (Vector Eurobio/Abcys, Courtaboeuf, France) and the fast red substrate (Dako France SAS).

\section{Culture of Fibroblasts and TGF- $\beta$ Stimulation}

Control pulmonary fibroblasts were obtained from LGC standards human cell lines (LGC Standards SARL, Molsheim, France). Fibrotic human fibroblasts were isolated from lung tissue from patients with IPF and cultured (IPF fibroblasts) as previously described. ${ }^{14}$ Briefly, lung tissue samples were obtained by open lung biopsy. Fibroblasts were cultured with Dulbecco's modified Eagle's medium (DMEM) with 10\% fetal calf serum supplemented with antibiotics. Human lung fibroblasts were cultured until passage six and were incubated in the presence or not of TGF- $\beta(2-30 \mathrm{ng} / \mathrm{ml}, \mathrm{R} \& \mathrm{D}$ Systems Europe, Lille, France) for 6-48 h.

\section{Reverse Transcription and Quantitative Real-time PCR}

Total RNA was isolated from fibroblasts using Trizol reagent (Invitrogen/Life Technologies SAS, Saint Aubin, France), according to the manufacturer's directions. Double-stranded cDNAs were synthesized and amplified as previously described. ${ }^{15}$ Real-time PCR was performed in the LightCycler system with SYBR Green detection (Roche Applied Science, Meylan, France). The following run protocol for PN-1, PAI-1, collagen $1 \alpha 2$, collagen $3 \alpha 1$, and fibronectin was used: denaturation: $95^{\circ} \mathrm{C}, 5 \mathrm{~min}$; and amplification and quantitation (40 cycles): $60^{\circ} \mathrm{C}, 10 \mathrm{~min} ; 72^{\circ} \mathrm{C}, 30 \mathrm{~min}$. Fluorescence of the samples was monitored continuously while the temperature was increased from 62 to $95^{\circ} \mathrm{C}$ at a linear transition rate of $0.1{ }^{\circ} \mathrm{C} / \mathrm{s}$, and arrested by a final cooling step at $45^{\circ} \mathrm{C}$. PN-1 primers were: forward $5^{\prime}$-GGGCAYCCTGGGCTAMACTG A- $3^{\prime}$ and reverse $5^{\prime}$-TGCTCTTGCTGGGGCTGGA-3'; PAI-1 primers were: forward $5^{\prime}$-GCACAACCCCACAGGAAC- $3^{\prime}$ and reverse $5^{\prime}$-TGCTTCAAACTTCTCTCCCAG- $3^{\prime}$; collagen $1 \alpha 2$ primers were: forward $5^{\prime}$-CGAGGTAGTCTTTCAGCAA CACAGT- $3^{\prime}$ and reverse $5^{\prime}$-TTGAGACTCAGCCACCCAGA GT-3'; collagen $3 \alpha 1$ primers were: forward 5-AGCTGGAAAG AGTGGTGACAG-3' and reverse 5'-CCTTGAGGACCAGGA GCAC-3'; and fibronectin primers were: forward $5^{\prime}$-CTGGC CGAAAATACATTGTAAA-3' and reverse $5^{\prime}$-CCACAGTCGG GTCAGGAG-3'. LightCycler run protocol for GAPDH was as follows: denaturation: $95^{\circ} \mathrm{C}, 5 \mathrm{~min}$; and amplification and quantitation $\left(40\right.$ cycles): $65^{\circ} \mathrm{C}, 10 \mathrm{~min} ; 72^{\circ} \mathrm{C}, 20 \mathrm{~min}$. GAPDH primers were: forward $5^{\prime}$-GTGAAGGTCGGAGTCA CG- $3^{\prime}$ and reverse $5^{\prime}$-GGTGAAGACGCCAGTGGACTC- $3^{\prime}$. Transcripts levels were normalized to GAPDH mRNA.

\section{Immunoblot Analysis}

Culture media and cell lysates were collected from TGF- $\beta$ stimulated and IPF fibroblasts. Proteins were extracted by cell lysis in ice-cold lysis buffer $(50 \mathrm{mM}$ Tris- $\mathrm{HCl}, \mathrm{pH} 7.5$, $150 \mathrm{mM} \mathrm{NaCl} ; 3 \mathrm{mM}$ EDTA, $0.1 \%$ SDS, $1 \%$ Nonidet P-40) containing a protease inhibitor cocktail (Sigma-Aldrich, Saint-Quentin Fallavier, France) and centrifuged at $5000 \mathrm{~g}$ for $5 \mathrm{~min}$ at $4{ }^{\circ} \mathrm{C}$; samples were then analyzed by immunoblotting. Protein concentrations were measured using a BCA Pierce protein assay (Interchim, Montluçon, France). Proteins were solubilized with $2 \%$ SDS and separated by 
electrophoresis on $10 \%$ acrylamide SDS gel. Immunoblots were performed using the monoclonal anti-PN-1 IgG $(1 \mathrm{~F} 6 ; 2 \mu \mathrm{g} / \mathrm{ml})$, a monoclonal anti-fibronectin IgG $(1 \mu \mathrm{g} / \mathrm{ml}$; Santa Cruz/CliniSciences, Nanterre, France), and a monoclonal anti-GAPDH $(1 \mu \mathrm{g} / \mathrm{ml}$; Abcam) followed by horseradish peroxydase-conjugated secondary antibodies (Jackson ImmunoResearch Europe Ltd, Suffolk, UK) at a 1:50000 dilution. Immunoreactivity was visualized by chemiluminescence (ECL; Amersham/VWR International, Fontenaysous-bois, France).

\section{Thrombin Assay}

Confluent control fibroblasts were serum-starved overnight and incubated for $24 \mathrm{~h}$ with serum-free medium containing $10 \mathrm{ng} / \mathrm{ml}$ of TGF- $\beta$. Confluent IPF fibroblasts were only serum-starved overnight and incubated for $24 \mathrm{~h}$ with serumfree medium. At the end of the incubation, conditioned media (CM) were harvested for analysis. CM were incubated with $0.2 \mathrm{nM}$ human $\alpha$-thrombin (purified as previously described ${ }^{16}$ ) for $30 \mathrm{~min}$ at $37^{\circ} \mathrm{C}$ in the presence or absence of a blocking polyclonal anti-PN-1 $\operatorname{IgG}(100 \mu \mathrm{g} / \mathrm{ml})^{17}$ or a blocking monoclonal anti-PAI-1 IgG $(100 \mu \mathrm{g} / \mathrm{ml})$ (MA-33B8-307; Molecular Innovations/Gentaur Europe
BVBA, Kampenhout, Belgium). At the end of incubation, the thrombin chromogenic substrate S2238 (Cryopep, Montpellier, France) was added at a final concentration of $0.2 \mathrm{mM}$, and changes in the absorbance were recorded at $405 \mathrm{~nm}$. Residual thrombin activity is expressed as the ratio of the activity measured in the presence of the CM derived from controls, TGF- $\beta$-stimulated, or IPF fibroblasts to the activity measured in the presence of DMEM medium culture $\times 100$. The experimental procedure realized as described above was also performed directly on cultured cells.

\section{Plasmid and siRNA Transfection}

The pcDNA3 vectors containing the coding sequence for the wild-type $\mathrm{PN}-1$, the inactive mutant $\mathrm{PN}-1$ (mutated active site), or the variant of $\mathrm{PN}-1$ in which the heparin site is nonfunctional (mutated glycosaminoglycans (GAG) binding site) were produced as previously described. ${ }^{18}$ Control fibroblasts were transfected with an empty pcDNA3 vector (mock vector) or vectors containing the different $\mathrm{PN}-1$ coding sequences by using the XtremGene kit (Roche Applied Science) according to the manufacturer's instructions. Fibroblasts were assayed after $48 \mathrm{~h}$ of transfection to quantify PN-1, collagen I, and fibronectin expression. a

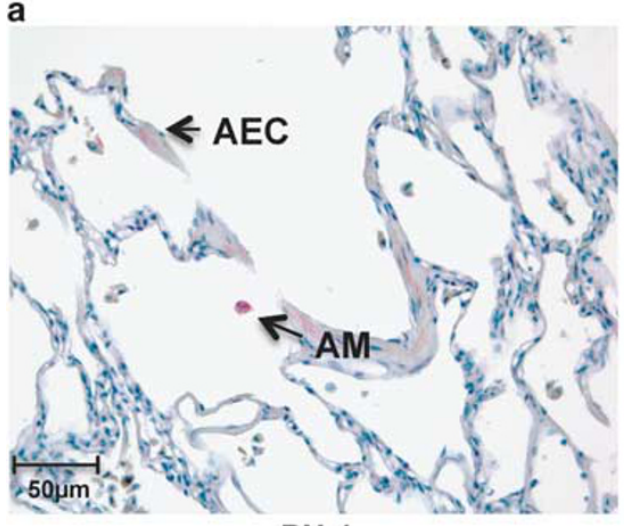

b

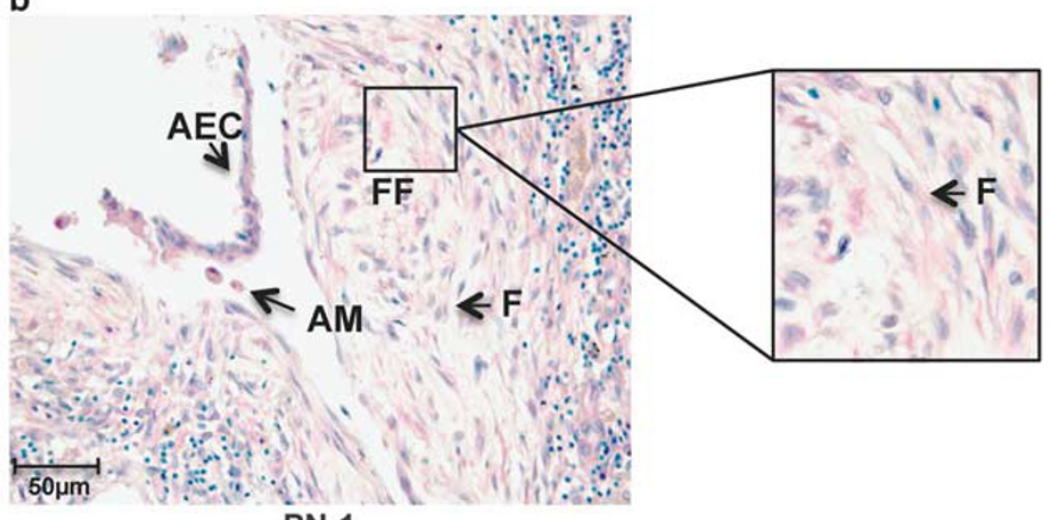

PN-1

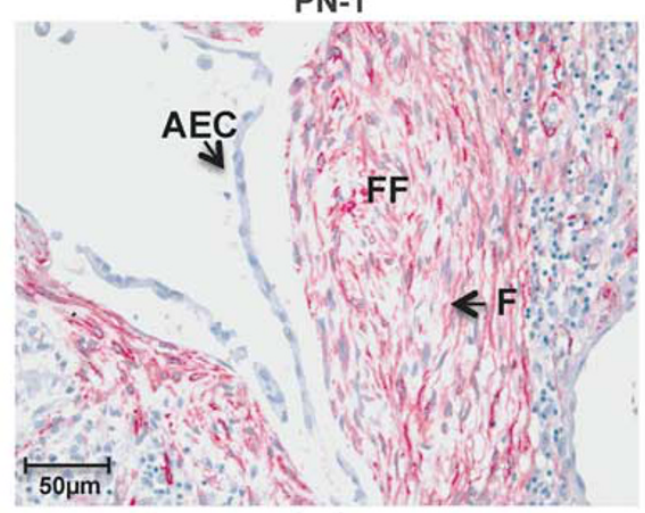

Actin

Figure 1 PN-1 localization in control lungs or lungs from patients with IPF. Human lung sections from controls (a) or patients with IPF (b) were analyzed by $\mathrm{PN}-1$ immunostaining. Sections were incubated with a monoclonal anti-PN-1 antibody, revealed by using the Vectastain ABC-alkaline phosphatase kit. Macroscopic analysis allowed PN-1 localization in lung sections at magnifications $\times 20$ and $\times 40$. Alveolar macrophages (AM), fibroblasts (F) in a fibroblastic focus (FF), and alveolar epithelial cells (AEC) were identified by morphological analysis. 
The following predesigned annealed siRNA were chosen for PN-1 silencing: sense sequence 5'-GGUUUUCAAUCAGAUUGAGTT- $3^{\prime}$ and antisense sequence $5^{\prime}$-CACAAUCUGAUUGAAAACCTT- $3^{\prime}$. The predesigned annealed irrelevant siRNA from Eurogentec was used as negative control. The duplexes were introduced into confluent IPF fibroblasts using lipofectamine reagents according to the manufacturer's instructions (Invitrogen/Life Technologies SAS). Cells were assayed after $96 \mathrm{~h}$ of transfection to verify $\mathrm{PN}-1$ extinction and quantify collagen I and fibronectin expression.

\section{Statistical Analysis}

Results are shown as means \pm s.e.m. The Student's t-test (cell culture experiments) or the Mann and Whitney's test (patients experiments) were used to analyze all experimental results, and $P<0.05$ was considered significant.

\section{RESULTS}

\section{PN-1 Localization in Normal and Pathological Human} Lung

Control lung sections showed an immune-reactivity for PN-1 in alveolar inflammatory cells, in particular in alveolar macrophages (AM) and in alveolar epithelial cells (Figure 1a). PN-1 immunostaining in lung samples from patients with IPF demonstrated additional prominent immunolocalization of $\mathrm{PN}-1$ in the fibroblastic focus (FF) clearly associated with fibroblasts as revealed by the positive $\alpha$-smooth muscle actin staining (Figure $1 \mathrm{~b}$ ).
Quantification of PN-1 mRNA in fibrotic and control lung tissues showed a two-fold increase of $\mathrm{PN}-1$ expression in lung tissues from patients with IPF compared with controls (Figure 2a).

Immunoblot analysis of human BAL fluid (BALF) from patients with IPF revealed a single band of $45 \mathrm{kDa}$ corresponding to native $\mathrm{PN}-1$ that was weakly detectable in controls (Figure 2b). Additional high-molecular-weight bands could be observed in BALF only in non-reducing conditions, indicating the presence of complexes formed between $\mathrm{PN}-1$ and other molecules via disulfide bridges. The dissociation of these higher bands under reducing conditions clearly demonstrates that they did not correspond to PN-1-protease complexes formed via the classical acyl-enzyme bond.

\section{PN-1 Expression in Human Control and Fibrotic Pulmonary Fibroblasts}

As shown in Figure 3a, PN-1 mRNA was detected in control cultured pulmonary fibroblasts at confluence. A significant increased expression of PN-1 mRNA (3.5-fold increase) was observed in fibroblasts cultured from patients with IPF (IPF fibroblasts), supporting the data obtained with the lung tissue extracts. PAI-1 was also expressed by pulmonary fibroblasts, and its expression was also increased in IPF fibroblasts (Figure 3a). The profibrotic phenotype of IPF fibroblasts was supported by the markedly increased expression of messengers of MEC components, such as collagen $1 \alpha 2$ (2-fold increase), collagen $3 \alpha 1$ (4-fold increase), and fibronectin (4.5-fold increase) (Figure 3a).
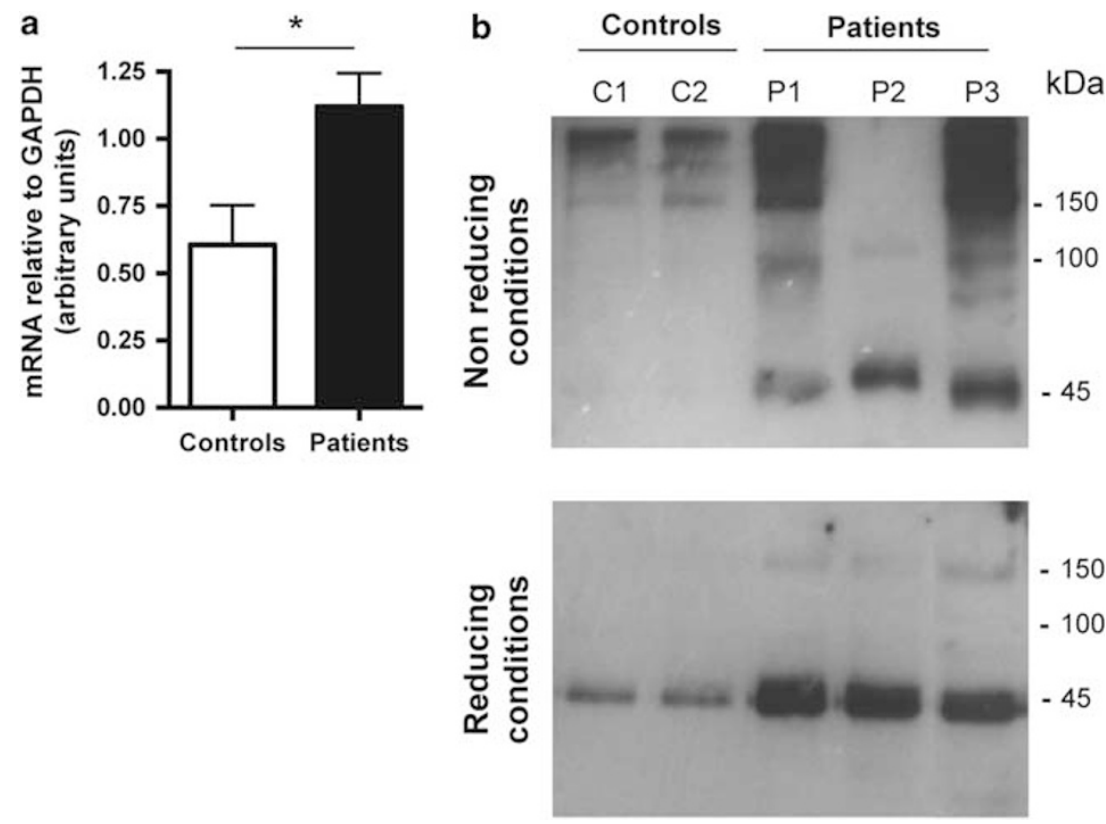

Figure 2 PN-1 is overexpressed in lung extracts and bronchoalveolar lavage (BAL) from patients with IPF. (a) PN-1 mRNA was extracted from lung tissue in controls and IPF patients, measured by qPCR and normalized with a housekeeping gene (GAPDH). Results are presented as means \pm s.e.m with $n=7$ for controls and IPF patients ( ${ }^{*} P<0.05$ significantly different from controls). (b) BALs were analyzed by immunoblot using the monoclonal anti-PN-1 antibody in non-reducing and reducing conditions. 
We can also observe an increased expression of PN-1 in IPF fibroblasts compared with control fibroblasts by immunoblot (Figure $3 \mathrm{~b}$ ), supporting the data obtained with the mRNA analysis. Indeed, the results showed that increased expression of PN-1 was largely detected in the CM of IPF fibroblast cultures, whereas little $\mathrm{PN}-1$ was detected in the $\mathrm{CM}$ of control fibroblast cultures. $\mathrm{PN}-1$ expressed by control fibroblasts remained essentially bound to cell surface because of the well-known high affinity of PN-1 to cell surface GAG. Altogether, these results indicate that when the fibroblast surface was saturated by $\mathrm{PN}-1$, the excess of $\mathrm{PN}-1$ expressed in fibrotic fibroblasts was released from the cell surface and found in the CM.

\section{TGF- $\beta$ Upregulates PN-1 Expression in Human Pulmonary Fibroblasts}

The effect of the well-known profibrotic cytokine TGF- $\beta$ on $\mathrm{PN}-1$ expression was analyzed at both the mRNA and protein levels, by qRT-PCR and immunoblotting. Exposure of control fibroblasts to TGF- $\beta$ induced the upregulation of PN-1 mRNA in a dose-dependent manner. The maximal stimulatory effect was reached at $10 \mathrm{ng} / \mathrm{ml}$ TGF- $\beta$, PN-1 mRNA being increased by 11-fold compared with unstimulated fibroblasts (Figure 4a). In time course experiments, the upregulation of $\mathrm{PN}-1$ messenger was maximal after $24 \mathrm{~h}$ of incubation with $10 \mathrm{ng} / \mathrm{ml}$ TGF- $\beta$ (Figure $4 \mathrm{~b}$ ).

TGF- $\beta$ also upregulated PN-1 protein expression by control fibroblasts in a dose (Figure 4c) and time (Figure 4d) dependent manner, the maximal stimulatory effect being observed at $10 \mathrm{ng} / \mathrm{ml}$ TGF- $\beta$ for $24 \mathrm{~h}$. Increased PN-1 was found in large amount in the CM from TGF- $\beta$-stimulated fibroblasts, indicating that PN-1 was secreted by TGF- $\beta$-stimulated fibroblasts as observed with IPF fibroblasts.

\section{PN-1 from Fibrotic Fibroblasts Inhibits Thrombin}

We examined whether increased PN-1 expressed by fibrotic fibroblasts was functional by investigating its ability to inhibit the amidolytic activity of purified thrombin. CM from unstimulated control fibroblasts had no effect on thrombin activity (Figure 5a). In contrast, thrombin residual activity dropped to $29 \pm 5 \%$ and $42 \pm 3 \%$ of its initial activity, when incubated with CM from TGF- $\beta$-stimulated and IPF
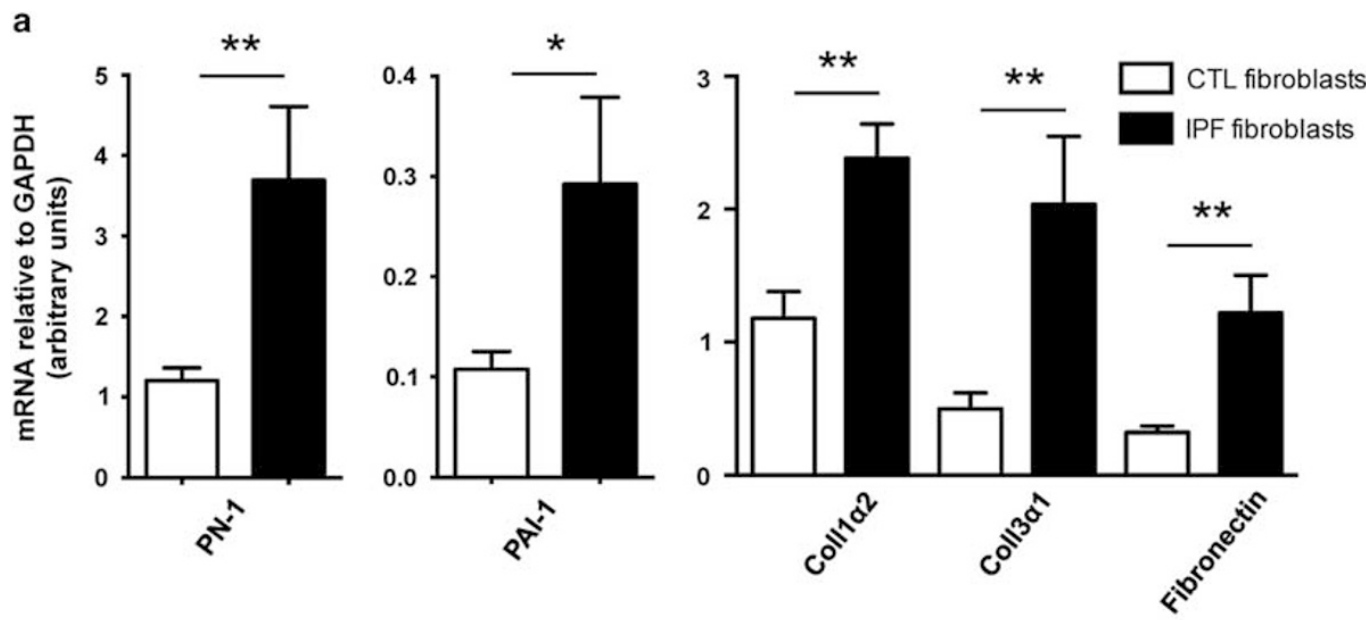

b
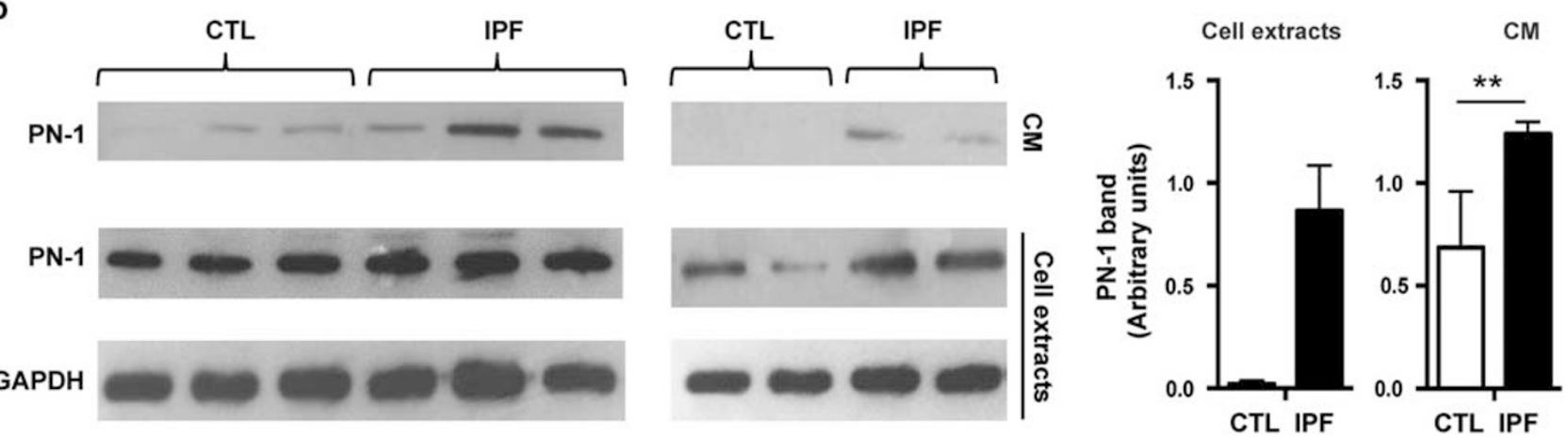

Figure $3 \mathrm{PN}-1$ is overexpressed in lung fibroblasts from patients with IPF. mRNA and protein were extracted from control (CTL) fibroblasts and IPF fibroblasts. (a) PN-1, PAI-1, collagen $1 \alpha 2$, collagen $3 \alpha 1$, and fibronectin mRNA were measured by qPCR and normalized with a housekeeping gene (GAPDH). Results are presented as means \pm s.e.m with $n=4$ for CTL and IPF fibroblasts ( ${ }^{*} P<0.01$ and ${ }^{*} P<0.05$ vs $C T L$ fibroblasts). (b) PN-1 expression in cell extracts and conditioned media (CM) were analyzed by immunoblotting using anti-PN-1 and anti-GAPDH antibodies, and results were quantified by densitometric analysis. On the left, immunoblots are shown, and on the right, densitometric analysis of the corresponding results are shown as means \pm s.e.m with $n=5$ for CTL and IPF. ( ${ }^{* *} P<0.01$ vs control fibroblasts). 


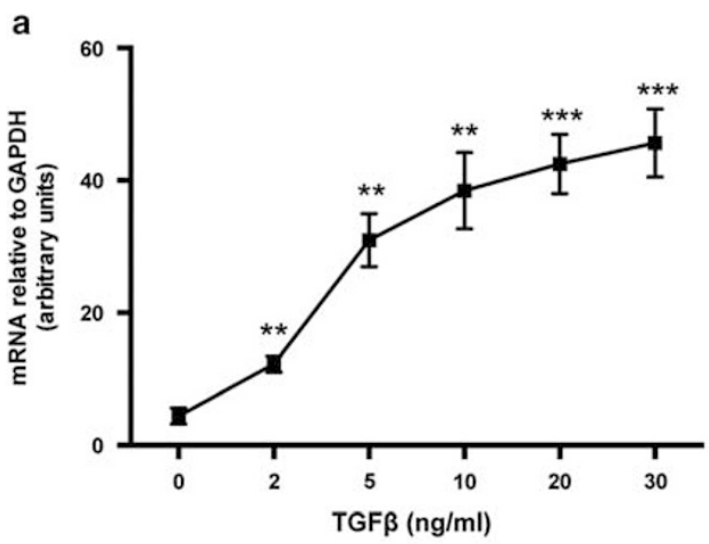

C

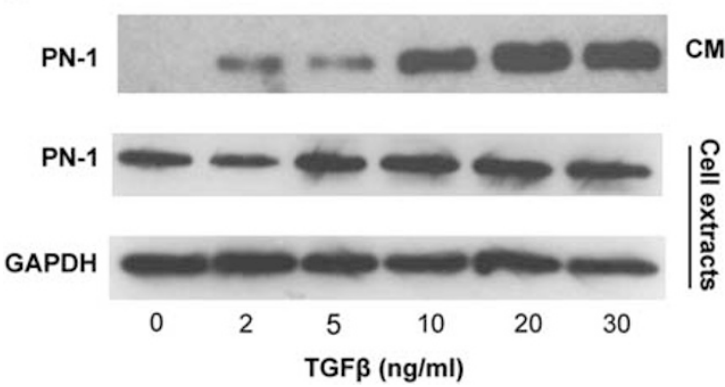

b

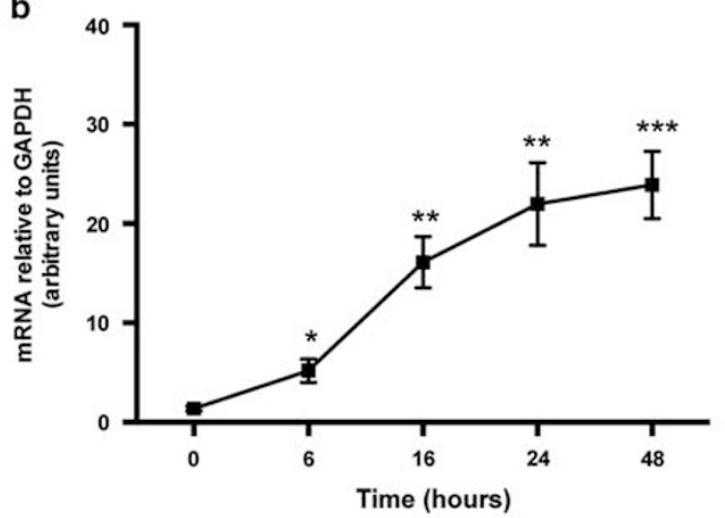

d

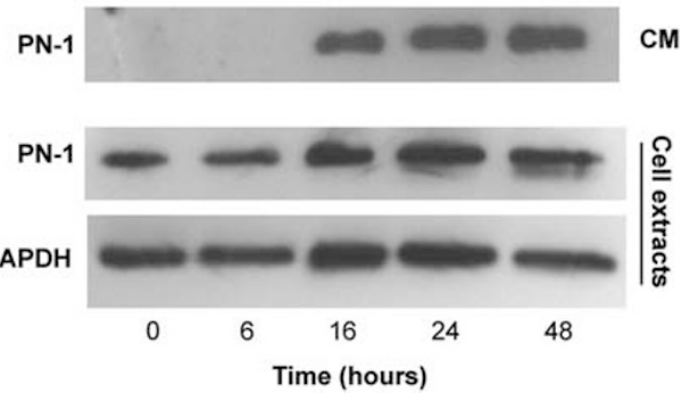

Figure 4 TGF- $\beta$ upregulates PN-1 expression in control lung fibroblasts. mRNA and proteins were extracted from fibroblasts as described in Materials and Methods. (a, c) Fibroblasts were stimulated by increasing doses of TGF- $\beta(0-30 \mathrm{ng} / \mathrm{ml})$ for $24 \mathrm{~h}$ or $(\mathbf{b}, \mathbf{d})$ by $10 \mathrm{ng} / \mathrm{ml} \mathrm{TGF}-\beta$ for $0-48 \mathrm{~h}$. (a, b) PN-1 mRNA of was measured by qPCR (means \pm s.e.m; $n=3$ independent experiments; ${ }^{*} P<0.05 ;{ }^{* *} P<0.01 ;{ }^{* * *} P<0.001$ vs unstimulated cells). (c, d) Cell extracts and conditioned media (CM) were analyzed by immunoblotting using anti-PN-1 and anti-GAPDH antibodies.

fibroblasts, respectively, indicating the presence of a thrombin inhibitor in these CM (Figure 5a). To verify that this thrombin inhibitor was $\mathrm{PN}-1$, the same experiments were performed in the presence of a blocking anti-PN-1 antibody. This blocking antibody significantly abolished thrombin inhibition by the CM derived from both IPF and TGF- $\beta$ stimulated fibroblasts. Such an effect was not observed with an irrelevant IgG or a blocking anti-PAI-1 antibody (Figure 5a). The same kind of result was obtained when analyzing thrombin catalytic activity directly on the cell surface of the different types of fibroblasts (Figure 5b). Indeed, both IPF and TGF- $\beta$-stimulated fibroblasts acquired a potent anti-thrombin effect that was reversed in the presence of the blocking anti-PN-1 antibody (Figure 5b). Together, these results indicate that the increase of PN-1 expression observed in fibrotic fibroblasts results in the accumulation of active PN-1 both at the cell surface and in the culture medium.

\section{PN-1 Overexpression in Pulmonary Fibroblasts Induces Fibronectin Expression}

To establish whether PN-1 could influence the expression of ECM proteins, we induced an overexpression of $\mathrm{PN}-1$ in control fibroblasts by transient transfection with a vector containing the complete $\mathrm{PN}-1$ coding sequence. A marked increase in the expression of $\mathrm{PN}-1$ was found compared with cells transfected with an empty vector (Figure 6a). Overexpression of PN-1 was accompanied by a two-fold increase in the expression of fibronectin, whereas it had no significant effect on the expression of collagen $1 \alpha 2$ and collagen $3 \alpha 1$ transcripts (Figure 6a). Conversely, a two-fold decrease in the expression of fibronectin was observed when PN-1 expressed by fibrotic fibroblasts from IPF patients was silenced by siRNA transfection (Figure 6b), whereas no change was observed in the expression of collagen $1 \alpha 2$ and collagen $3 \alpha 1$ transcripts.

PN-1 and the majority of the other serpins bind to their targets by using a substrate recognition sequence contained in an exposed reactive center loop. They also have a GAG binding site that causes the modulation by GAG of the activity of several serpins. To verify that fibronectin overexpression depended on the expression of functional PN-1, we transfected control fibroblasts with a vector containing, respectively, the $\mathrm{PN}-1$ coding sequence with a mutated reactive center loop (RS mutant $\mathrm{PN}-1$ ) or with a mutated GAG-binding site (HS mutant PN-1). Both mutated PN-1 molecules failed to significantly induce fibronectin overexpression (Figure 6a), indicating that both the antiprotease activity of PN-1 and its GAG-binding site are required for such an effect. 

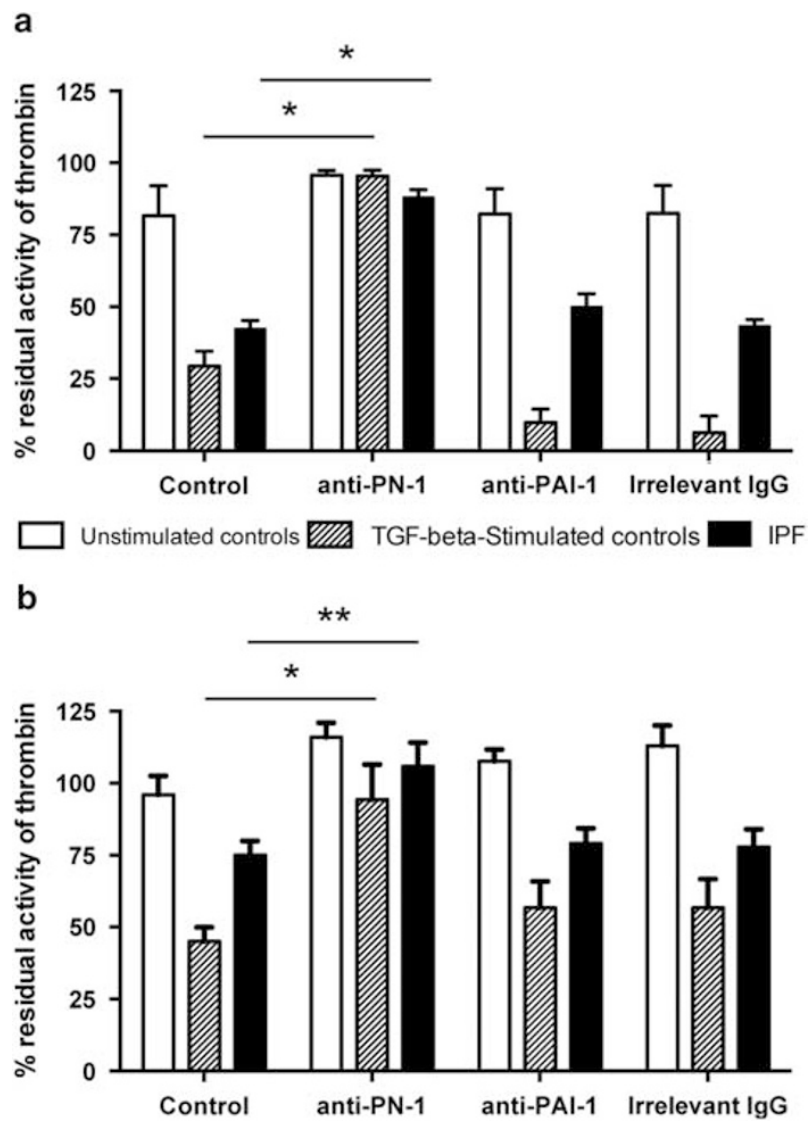

Figure 5 Inhibition of thrombin catalytic activity by PN-1 present in lung fibroblasts. Thrombin catalytic activity was measured after incubation (a) with conditioned media from unstimulated control fibroblasts, TGF- $\beta$-stimulated fibroblasts, or IPF fibroblasts or (b) on cell surface of unstimulated control fibroblasts, TGF- $\beta$-stimulated fibroblasts, or IPF fibroblasts. Incubations were performed in the presence or absence of a blocking anti-PN-1 IgG or anti-PAl-1 IgG or an irrelevant IgG. Results are presented as means \pm s.e.m of three independent experiments, each performed in triplicate $\left({ }^{*} P<0.05 ;{ }^{*} P<0.01 \mathrm{vs}\right.$ respective controls).

\section{DISCUSSION}

IPF is characterized not only by an accumulation of excess fibrous material in the lung resulting from an excessive production and deposition of ECM on the one hand but also by decreased proteolysis and clearance of ECM on the other hand. The tissue balance of protease and antiprotease activities is therefore a cornerstone of IPF pathogenesis. The roles of plasminogen, plasminergic enzymes, and their regulators in the development of IPF have been well documented. High levels of PAI-1 have been observed in IPF and are associated with poor clinical outcomes. ${ }^{2,19}$ We demonstrated in this study that $\mathrm{PN}-1$, another serpin, was also abnormally highly expressed in the fibrotic lung tissue and detected in BALF from patients with IPF. Until now, no data were available concerning the role of $\mathrm{PN}-1$ in the pulmonary fibrosis. PN-1 is the most potent tissue inhibitor of thrombin. Our results clearly showed that both $\mathrm{PN}-1$ present on the fibroblast surface and PN-1 secreted by lung fibroblasts were able to block thrombin activity, whereas PAI-1 had no such inhibitory effect, confirming that PAI-1 is a weak inhibitor of thrombin. ${ }^{20}$ Such data suggest that the regulation of thrombin, and therefore coagulation in the lungs, might be ensured by PN-1 rather than PAI-1. However, the respective implications of PN-1 and PAI-1 in the pathogenesis of IPF remain unknown. If degradation of ECM and fibrinolysis are the dominant processes involved in lung remodeling occurring during IPF, PN-1 and PAI-1 should exhibit complementary roles. In contrast, if fibrin formation induced by thrombin and pleiotropic cellular effects of thrombin are other dominant processes, therefore PN-1 should play a major role in the pathogenesis of IPF.

In the normal situation, BALF is known to exhibit fibrinolytic activity due to the presence of uPA. The role of uPA is to clear fibrin formed after extravasation of plasma proteins into the alveolar space. ${ }^{21}$ During IPF, increased levels of inhibitors, in particular PAI-1, suppress this fibrinolytic activity. $^{2}$ Our data show, for the first time, that there is also an abnormal high level of PN-1 in BALF from patients with IPF. PN-1 has been previously shown to limit fibrinolysis within a blood clot via its ability to inhibit plasminergic enzymes. ${ }^{22}$ In the blood, the regulation of the plasminergic system by serpins, such as PAI-1, PN-1, or $\alpha 2$-antiplasmin, depends on their access to the fibrin clot. Here, in the lung, we are in a situation where these serpins may have complementary actions to regulate the plasminergic system taking place in the lung tissue.

In lung tissue from patients with IPF, PN-1 immunostaining was not only observed in fibroblasts but also in large immune cells, the AM. This is in agreement with previous data showing that PN-1 is expressed by blood monocytes and monocyte-derived macrophages. ${ }^{23}$ However, a major part of PN-1 immunostaining was detected in lung fibroblasts, in particular in fibrotic areas. $\mathrm{PN}-1$ is known to be expressed by fibroblasts from many different tissue types, including foreskin fibroblasts, ${ }^{6}$ skin fibroblasts, ${ }^{8}$ or mouse embryonic fibroblasts. ${ }^{24}$ In human foreskin fibroblasts, PN-1 expression has been shown to be upregulated by interleukin-1. ${ }^{25} \mathrm{We}$ showed here that TGF- $\beta$, a potent profibrogenic cytokine, could also upregulate PN-1 expression in lung fibroblasts. Such upregulation of PN-1 expression induced by TGF- $\beta$ has been shown in many different cultured cells, including neuroblastoma, ${ }^{26}$ myotubes, ${ }^{27}$ renal epithelial cells or cortical collecting duct cells, ${ }^{28}$ and vascular smooth muscle cells. ${ }^{29}$ PAI-1 is also overexpressed by pulmonary fibroblasts stimulated by TGF- $\beta,{ }^{30}$ and functional analysis of both PAI-1 and PN-1 promoters have identified a regulatory region for TGF- $\beta$ induction, involving Smad complex binding. ${ }^{29}$ The possibility that upregulation of $\mathrm{PN}-1$ expression is a common process in fibrosis whatever the tissue affected remains to be determined.

PN-1 was present not only in the CM from fibroblast cultures but also in cell extracts. In contrast, PAI-1 is a 

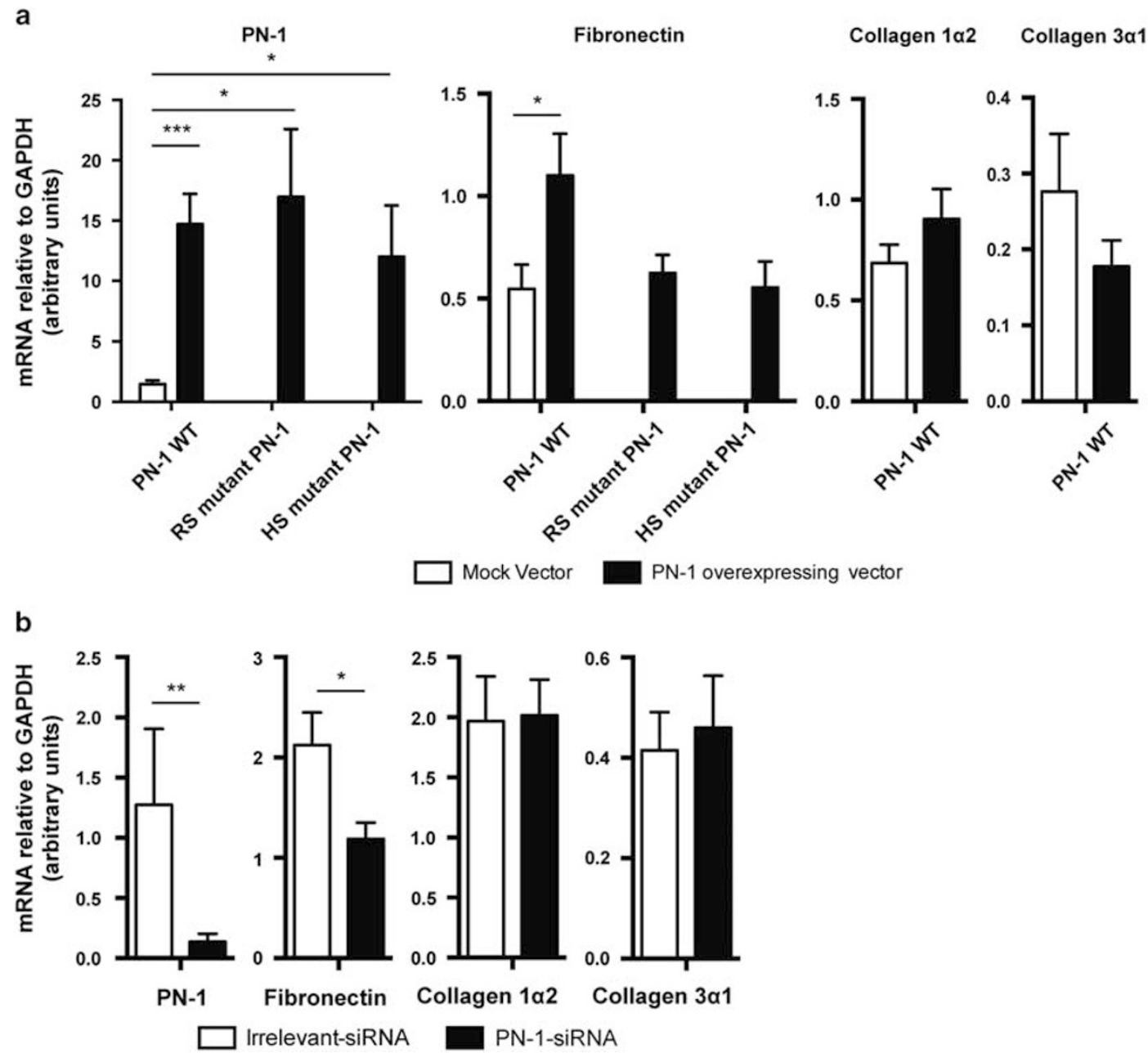

Figure 6 Modulation of PN-1 expression in lung fibroblasts regulates fibronectin expression. Control fibroblasts were transiently transfected with a WT PN-1 overexpressing vector or mutated PN-1 overexpressing vectors: reactive center loop mutant (RS) or GAG binding site mutant (HS). Negative controls were performed with a mock vector. (a) PN-1, fibronectin collagen $1 \alpha 2$, and collagen $3 \alpha 1 \mathrm{mRNA}$ were analyzed by qPCR and normalized with the housekeeping gene (GAPDH). Results are presented as mean \pm s.e.m of four independent experiments, each performed in duplicate $\left({ }^{*} P<0.05\right.$; ${ }^{* * *} P<0.001$ vs transfection with the mock vector). (b) IPF fibroblasts were transfected with irrelevant small interfering RNA (siRNA) or siRNA for PN-1 (PN-1-siRNA). PN-1, collagen 1 $\alpha 2$, collagen $3 \alpha 1$, and fibronectin mRNA were analyzed by qPCR and normalized with GAPDH. Results are presented as mean \pm s.e.m of four independent experiments, each performed in duplicate $\left({ }^{*} P<0.05\right.$; ${ }^{* *} P<0.01$ vs transfection with the mock vector).

protein directly secreted into the culture medium and therefore poorly detectable in cell extracts. Such differences in cellular localization between PAI-1 and PN-1 can be explained by the fact that PN-1 has a higher affinity for GAG than PAI-1. Indeed, $\mathrm{PN}-1$ has been shown to remain essentially associated with cell surface GAG and to be released from the cell surface by polysaccharides, such as heparin ${ }^{31}$ or fucoidans. ${ }^{32}$ Thus, in physiological conditions, $\mathrm{PN}-1$ and PAI-1 are present in different compartments and therefore may target different proteases. We found that part of the PN-1 induced by TGF$\beta$-stimulated and IPF fibroblasts was detected in CM in large amounts because of a saturation of the fibroblast surface under these fibrotic conditions. These variations in the localization of $\mathrm{PN}-1$ suggest that its role may become important under conditions of stress, as here under fibrotic conditions.

The simultaneous increased expression of $\mathrm{PN}-1$ and ECM proteins in TGF- $\beta$-stimulated and IPF fibroblasts raised the question of a potential direct modulation of ECM production by the abnormally high level of PN-1. We indeed showed that upregulation of PN-1 expression induced by transient transfection in control pulmonary fibroblasts was accompanied by an upregulation of fibronectin expression, and conversely $\mathrm{PN}-1$ silencing induced by transfection with a PN-1-siRNA in fibrotic pulmonary fibroblasts from IPF patients was accompanied by a downregulation of fibronectin expression. These results suggest that $\mathrm{PN}-1$ upregulation is not only a consequence of fibrosis but also contributes to fibronectin accumulation. No effect on fibronectin expres- 
sion was observed when using mutated forms of $\mathrm{PN}-1$, confirming a direct relationship between $\mathrm{PN}-1$ and fibronectin expression in fibrotic fibroblasts, and that PN-1 requires both its antiprotease activity and its capacity to bind GAG to affect fibronectin expression. Upregulation of PN-1 expression has previously been shown to induce increased collagen transcript levels in mouse embryonic fibroblasts, but no data were provided for fibronectin transcripts. ${ }^{8}$ In contrast, our results did not show any significant change for collagen expression in lung fibroblasts overexpressing PN-1. We can assume that, according to the studied tissue type, $\mathrm{PN}-1$ overexpressed by fibroblasts may be involved in the upregulation of certain MEC proteins. PN-1 may induce different signaling pathways, leading to a differential regulation of ECM proteins, depending on the studied cell type.

PN-1 has been shown to inhibit furin, ${ }^{33}$ an ubiquitous proprotein convertase involved in the proteolytic processing of a wide range of precursor proteins, including adhesion molecules and various metalloproteinases known to be involved in ECM degradation. ${ }^{34}$ Therefore PN-1 may also have a role in the modulation of ECM expression by its capacity to inhibit such regulatory proteases.

Our data confirm that PN-1 has a role in lung homeostasis. PN-1 has indeed recently been evaluated as a susceptibility gene for another devastating lung disease: chronic obstructive pulmonary disease (COPD). ${ }^{10,35}$ Some PN-1 variants have been associated with lung function among patients with COPD and asthma. ${ }^{36}$ We thus propose that $\mathrm{PN}-1$ is a new important player in pulmonary fibrosis and hypothesize that the biological activity of $\mathrm{PN}-1$ could be of importance in the progression of the pathology. Such a role could be deleterious, regarding the impact of $\mathrm{PN}-1$ on the induction of ECM proteins such as fibronectin or, in contrast, beneficial in view of the impact of $\mathrm{PN}-1$ on thrombin activity. The novel identification of $\mathrm{PN}-1$ as a new player in IPF development might lead to a better understanding of the lung remodeling occurring during this disease and thus be helpful for designing new therapeutic approaches.

\section{ACKNOWLEDGMENTS}

This work was supported by INSERM (Institut National de la Santé et de la Recherche Médicale) and by University Paris 7 Denis Diderot. Deborah François was the recipient of a fellowship from the Fondation pour la Recherche Médicale (FRM). The authors thank Dr Monique Dehoux for providing the bronchoalveolar lavage fluids from IPF patients.

\section{AUTHOR CONTRIBUTIONS}

DF and LV contributed to acquisition, analysis and interpretation of data; JM-S carried out immunohistochemical experiments and, with $B C$, contributed to material tools and tissue specimen; MJ-P and VA revised the manuscript; M-CB contributed to conception of experiments, design of the study, interpretation of data, drafting and revising the article. All authors have approved the submitted manuscript.

\section{DISCLOSURE/CONFLICT OF INTEREST}

The authors declare no conflict of interest.

1. Huntington JA. Serpin structure, function and dysfunction. J Thromb Haemost 2011;9(Suppl 1):26-34.

2. Kotani I, Sato A, Hayakawa $H$, et al. Increased procoagulant and antifibrinolytic activities in the lungs with idiopathic pulmonary fibrosis. Thromb Res 1995;77:493-504.

3. Senoo T, Hattori N, Tanimoto $\mathrm{T}$, et al. Suppression of plasminogen activator inhibitor-1 by RNA interference attenuates pulmonary fibrosis. Thorax 2010;65:334-340.

4. Sisson $\mathrm{TH}$, Hanson $\mathrm{KE}$, Subbotina $\mathrm{N}$, et al. Inducible lung-specific urokinase expression reduces fibrosis and mortality after lung injury in mice. Am J Physiol Lung Cell Mol Physiol 2002;283:L1023-L1032.

5. Eaton DL, Baker JB. Evidence that a variety of cultured cells secrete protease nexin and produce a distinct cytoplasmic serine proteasebinding factor. J Cell Physiol 1983;117:175-182.

6. Baker JB, Low DA, Simmer RL, et al. Protease-nexin: a cellular component that links thrombin and plasminogen activator and mediates their binding to cells. Cell 1980;21:37-45.

7. Gloor S, Odink K, Guenther J, et al. A glia-derived neurite promoting factor with protease inhibitory activity belongs to the protease nexins. Cell 1986;47:687-693.

8. Strehlow D, Jelaska A, Strehlow K, et al. A potential role for protease nexin 1 overexpression in the pathogenesis of scleroderma. J Cin Invest 1999;103:1179-1190.

9. Mansuy IM, van der Putten $H$, Schmid $P$, et al. Variable and multiple expression of Protease Nexin-1 during mouse organogenesis and nervous system development. Development 1993;119:1119-1134.

10. Demeo DL, Mariani TJ, Lange $C$, et al. The SERPINE2 gene is associated with chronic obstructive pulmonary disease. Am J Hum Genet 2006; 78:253-264.

11. Blobe GC, Schiemann WP, Lodish HF. Role of transforming growth factor beta in human disease. New Eng J Med 2000;342:1350-1358.

12. Quesnel C, Marchand-Adam S, Fabre A, et al. Regulation of hepatocyte growth factor secretion by fibroblasts in patients with acute lung injury. Am J Physiol Lung Cell Mol Physiol 2008;294:L334-L343.

13. Bouton $M C$, Venisse $L$, Richard $B$, et al. Protease nexin- 1 interacts with thrombomodulin and modulates its anticoagulant effect. Circ Res 2007;100:1174-1181.

14. Marchand-Adam S, Fabre A, Mailleux AA, et al. Defect of prohepatocyte growth factor activation by fibroblasts in idiopathic pulmonary fibrosis. Am J Respir Crit Care Med 2006;174:58-66.

15. Richard B, Pichon S, Arocas V, et al. The serpin protease nexin-1 regulates vascular smooth muscle cell adhesion, spreading, migration and response to thrombin. J Thromb Haemost 2006;4:322-328.

16. Bezeaud A, Denninger MH, Guillin MC. Interaction of human alphathrombin and gamma-thrombin with antithrombin III, protein $\mathrm{C}$ and thrombomodulin. Eur J Biochem/FEBS 1985;153:491-496.

17. Boulaftali $Y$, Adam F, Venisse $L$, et al. Anticoagulant and antithrombotic properties of platelet protease nexin-1. Blood 2010;115: 97-106.

18. Selbonne S, Azibani $F$, latmanen $S$, et al. In vitro and in vivo antiangiogenic properties of the serpin protease nexin-1. Mol Cell Biol 2012;32:1496-1505.

19. Sapru A, Curley MA, Brady S, et al. Elevated PAl-1 is associated with poor clinical outcomes in pediatric patients with acute lung injury. Intensive Care Med 2010;36:157-163.

20. van Meijer M, Smilde A, Tans G, et al. The suicide substrate reaction between plasminogen activator inhibitor 1 and thrombin is regulated by the cofactors vitronectin and heparin. Blood 1997;90: 1874-1882.

21. Bertozzi P, Astedt B, Zenzius L, et al. Depressed bronchoalveolar urokinase activity in patients with adult respiratory distress syndrome. New Eng J Med 1990;322:890-897.

22. Boulaftali Y, Ho-Tin-Noe B, Pena A, et al. Platelet protease nexin-1, a serpin that strongly influences fibrinolysis and thrombolysis. Circulation 2011;123:1326-1334.

23. Mansilla S, Boulaftali Y, Venisse L, et al. Macrophages and platelets are the major source of protease nexin-1 in human atherosclerotic plaque. Arterioscler Thromb Vasc Biol 2008;28:1844-1850. 
24. Li X, Herz J, Monard D. Activation of ERK signaling upon alternative protease nexin-1 internalization mediated by syndecan-1. J Cell Biochem 2006:99:936-951.

25. Guttridge DC, Lau AL, Cunningham DD. Protease nexin-1, a thrombin inhibitor, is regulated by interleukin-1 and dexamethasone in normal human fibroblasts. J Biol Chem 1993;268:18966-18974.

26. Vaughan PJ, Cunningham DD. Regulation of protease nexin-1 synthesis and secretion in cultured brain cells by injury-related factors. J Biol Chem 1993;268:3720-3727.

27. Mbebi $C$, Hantai $D$, Jandrot-Perrus $M$, et al. Protease nexin I expression is up-regulated in human skeletal muscle by injury-related factors J Cell Physiol 1999;179:305-314.

28. Wakida N, Kitamura K, Tuyen DG, et al. Inhibition of prostasin-induced $\mathrm{ENaC}$ activities by $\mathrm{PN}-1$ and regulation of $\mathrm{PN}-1$ expression by TGF-beta1 and aldosterone. Kidney Int 2006;70:1432-1438.

29. Gomez D, Kessler K, Borges LF, et al. Smad2-dependent protease nexin-1 overexpression differentiates chronic aneurysms from acute dissections of human ascending aorta. Arterioscler Thromb Vasc Biol 2013;33:2222-2232.

30. Keski-Oja J, Raghow R, Sawdey M, et al. Regulation of mRNAs for type-1 plasminogen activator inhibitor, fibronectin, and type I procollagen by transforming growth factor-beta. Divergent responses in lung fibroblasts and carcinoma cells. J Biol Chem 1988;263:3111-3115.

31. Richard B, Arocas V, Guillin MC, et al. Protease nexin-1: a cellular serpin down-regulated by thrombin in rat aortic smooth muscle cells. J Cell Physiol 2004;201:138-145.

32. Richard B, Bouton MC, Loyau S, et al. Modulation of protease nexin-1 activity by polysaccharides. Thromb Haemost 2006;95: 229-235.

33. Boulaftali Y, Francois D, Venisse L, et al. Endothelial protease nexin-1 is a novel regulator of $A$ disintegrin and metalloproteinase 17 maturation and endothelial protein $C$ receptor shedding via furin inhibition. Arterioscler Thromb Vasc Biol 2013;33: 1647-1654.

34. Nakayama K. Furin: a mammalian subtilisin/Kex2p-like endoprotease involved in processing of a wide variety of precursor proteins. Biochem J 1997;327(Pt 3):625-635.

35. Zhu G, Warren L, Aponte J, et al. The SERPINE2 gene is associated with chronic obstructive pulmonary disease in two large populations. Am J Resp Crit Care Med 2007;176:167-173.

36. Himes B, Klanderman B, Ziniti J, et al. Association of serpinE2 with asthma. Chest 2011;140:667-674. 